\title{
A Secure Communication using Smart Mobile and Cloud Process
}

\author{
Guntupalli Manoj Kumar, K.V.S.S.Hemanth, Akshay Kumar Sonkar
}

\begin{abstract}
Information storage and security is one of key areas where much research is been done in this digital world where we communicate the data over using third party devices such as cloud by using smart devices such as mobiles so the security is an quite challenging factor where we access our data across the globe and with the social media coming into factor for the storage and accessibility of the data so there are many risk factors coming into process so we need to implement a smart and secure system for the authentication threats so here in this paper we implement a smart system in which face recognition aunthication system is implement between the cloud and mobile activity which give more security in terms of data storage and communication and then we evaluate using different graphs and also analyses the attacks

Keywords: Cloud, Protocol Face Recognition, Mobile computing
\end{abstract}

\section{INTRODUCTION}

Various authentication frame-works are proposed then produced for the Smart-phones to verify private client data. Nonetheless, on account of shared assets like CC, verifying private data is certifiably not an ordinary undertaking because of its reliance on approximately coupled cloud assets. Sub sequently, implicit Smartphone confirmation systems are not adequate to give check and verification of outsider CC assets, since much of the time the client additionally needs to depend on the validation component given by the $\mathrm{CC}$ asset. For instance, when data is exchanged from Smart-phone to a cloud asset, the client needs to totally depend on the verification or protection system created by the specific asset.

These days, Smartphones are very much furnished with various validation instruments, for example, MFA, 2Factor Authentication (FA) and 3FA [ 18 - 20 ] . A 3 FA based Smartphone can give higher security to basic data. Be that as it may, it isn't important that cloud assets offer help for Multiple Factor Authentication or 3Factor Authentication based validation. Moreover, there is danger of a security

Revised Manuscript Received on September 22, 2019.

Mr.Guntupalli Manoj Kumar, Dept. of Computer Science and Engineering, SRMIST,Chennai, India, Manojkumar.na@ktr.srmuniv.in.

K.V.S.S.Hemanth, Dept. of Computer Science and Engineering,SRMIST,Chennai, India,kokavenkata_ko@srmuniv.edu,

Akshay Kumar Sonkar, Dept. of Computer Science and Engineering, SRMIST, Chennai, India, akshay.sonkar132@gmail.com.

break is more possible when such cloud asset is engaged with exchanging client basic data and approach worked in Smartphone assets. Then again, without the use of such access authorizations, those applications would neglect to complete fundamental assignments related with either day by day schedules or expert errands. Those dangers are not just constrained to Smartphones. These days, Smart gadgets, for example, telephone tabs and tablets have supplanted our normal Laptops and PCs. Besides, practically every space and segments which used those shrewd innovations to play out their ordinary or basic activities. The security hazard is huge when we also considertouch y s a ces like t h e Telecommunications, Military, Health, Defence and other legislative or private substances. [11-15] In view of the foretold understanding, different authentication structures, conventions are first proposed then created to give start for the finish protection, security and checks all substances and areas. In any case, there remains bounty to cover and investigate regarding protection and security in Smart- phones, Cloud Computing authentication systems. The reason for the investigation is to examine and report already present and essential security problems relating to Smart- phones and Cloud Computing Authentication Frameworks and Protocols.[16-17]

\section{CLOUD PROCESS}

\section{A. Analysation of the Performance of the Touch-Interaction Behaviour in an Active Smartphone Authentication}

A dependable and applicable examination of the client contact conduct for Smart-phone authentication had been researched where the static and dynamic highlights were verified for client touch characterisation. Many procedures were connected on the highlights for active authentication. About seventy one members information and approximately 135,000 touch activities were studied and then the judgment was passed on the operational execution. 


\section{B. Behavioural Biometrics Authentication for Smartphone}

Behavioural authentication and the dangers about it were talked about by A. Alzubaidi for example not only for the likes a stolen Smart- phones but also a secured Smart-phones. Various methodologies and systems, for example constant confirmation, were broke down fo conduct biometrics dependent on various procedures, datasets and appraisal approaches. The investigation finished up with different headings inside conduct biometric confirmation - Proposals included; th e convenience while concentrating on different qualities and client conduct estimation amid the application utilization.

\section{Energy Efficient Authentication for Smartphone Devices}

A vitality effective, quick and secure confirmation system is suggest $\mathrm{f}$ o $\mathrm{r}$ fascinating Smart-phone and distributed computing by P.Gasti. The creators guaranteed that the dynamic authentication and security protocols were not viable for Smart-telephones. The suggested work was assessed with physical try outs. The examination finishes up with the case of being the main investigation giving non stop and low - inactivity authentication. Be that as it may, the suggested protocols were just checked through step by step security investigation and are not bolstered with approval and confirmation utilizing verification or cryptographic convention approval strategies, instruments

$\begin{array}{lllllllllll}\mathrm{a} & \mathrm{n} & \mathrm{d} & \mathrm{s} & \mathrm{y} & \mathrm{s} & \mathrm{t} & \mathrm{e} & \mathrm{m} & \mathrm{s}\end{array}$

\section{Smartphone based Digital Identity Authentication}

The dient driven versatile Identity Management authentication protocol frame-work is suggested. There are a few confirmation and confinement in the proposed I $\mathrm{D}$

- security system m. Th e proposed arrangement had tended to just a couple of those zones, for example, summed up as general authentication frame-work and network integration. what's more, comes up short on the utilization of an standard verification check. The investigation distinguished the usage issues and downsides regarding clien tinclinations, QoS! (QoS!) service and management discovery. A authentication system was proposed, however incorporated no subtleties of the calculations utilized in the authentication conventions. In this manner, the IDM structure still requires a more extended time allotment to give total security approval.

\section{E. Payment of Android Mobile using Authentication Framework}

A3 Factor Authentication Smart-phone mobile payment for Android mobile was released. The plan highlights 3 Factor A u the $\mathrm{nt}$ i c ation confirmation mixing biometrics and One Time Password. The authentication feature depended on the HTTPS channel over Ad-hoc network showing the interface between the Smartphone and Android framework parts with the segments of the authentication system. There are numerous issues problems in this study. For example, the utilization of HTTPS isn't at all thought to be totally secure. Moreover, significant data was stored in the cell phone, which is exceptionally vulnerable if there should arise an occurrence of robbery or loss of the gadget. This prompts pantomime and offline brute force hacking. The structure has execution problems. The Ad-Hoc availability had used 15 practical activities between Reader, Payers and Database ,which results with lower execution of the structure. The structure had used AES / DES / 3DES which itself is powerless against cold-boot assaults. Those problems ought to be canvassed so as to think about this investigation for further execution.

\section{F. Factor Authentication Scheme for Bank Payment System}

In 2012, the authors suggested a Smart-phone b a s e d web based financial frameworkempowered with NFC prepared bank cards. The suggested framework included a Computer program furnished with 2 D barcode identification which is comprehensible in the client Smart-phone, which consequently contacts the Near Field Communication empowered bank card utilizing versatile NFC gear. The NFC-TAN strategy was utilized so as to contact the Near Field Communication empowered platinum card which enables the client to contact the gadget disconnected. The structure involves four stages, to be specific, login, examine, transaction and exchange. It shows the effortlessness of the proposed plan and the moderately couple of segments/ elements included. It was noticed that NFC- T A N w a defenseless against a n eavesdropping assault. 


\section{PROPOSED MODEL}

The proposition has delineated number of validation assaults, be that as it may, the creators neglected to clarify how their work is economical to those assaults. Next, a propelled versatile based qualification convention is displayed. Enhanced dynamic accreditation age conspire for insurance of client character in port table disc tribute muting $\mathrm{U} s \mathrm{~s} \mathrm{r}$ confirmation advance for information security in-between PDA, cloud. The session factors, T-1and $\mathrm{T}-2$, utilized in the convention are inaccurate, which makes it vulnerable to parallel session assaults. In light of the above understanding, different validation systems and conventions are proposed and created to give start to finish secure protection and check to all substances and spaces. Be that as it may, there remains bounty to cover and investigate regarding security and protection in smart- phones and cloud-computing validation structures. The motivation behind this investigation is for the examination of the existing records and essential security problems relating to smart-phones and for authentication of these cloud-computing frame- works. And remaining of the paper is composed as pursues: We give a concise foundati on on the nuts and bolts of Authentication Protocols, its Factors and Analysis Methods in Section 5. Point by point, exhaustive Literature Review is led in Section 2 by wrapping up numerous surveys of Smart- phone and Cloud Computing validation structures and conventions. Segment gives nitty gritty examination of the writing audit and features various difficulties regarding security and protection entanglements and peculiarities. Section 4 likewise introduces the rundown of the writing with the assistance of contrast and illustrative graphs. Section 7 finishes up this examination and talks about future headings in the light of the difficulties featured amid the investigations of the writing audit and the outline.

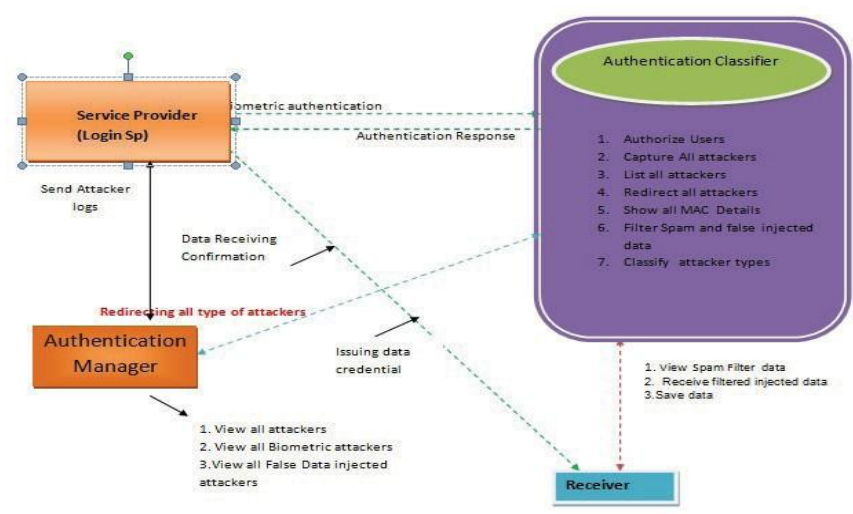

\section{MODULAR IMPLEMENTATIONS}

\section{A. Mobile Services Provider}

In this module, the Servicing provider is responsible for register using the Biometric au- then tic anion. The Biometric authentication its a way of logging in to project with face recognition or login with an image. If you are entered image and already exist images are getting match or biometric login is successful then service provider will get activate. Service provider browses the data File, and uploads their data files to the particular Receiver (Revive 1 , Revive 2, Revive 3, and Revive 4).

\section{B. Authentication Classifier}

The authentication classifier is responsible to scan their contents a Biometric Scan and Vulnerable word scan/ Span message scan.

\section{Biometric scan}

Authenticate the user with Biometric / image and then activate the Service provider Otherwise your image and existed image are not matched or Biometric authentication fails in pattern classifiers then related message and Image will be stores in pattern manager.

\section{E. Span message scan}

The pattern classifiers check if uploaded file contains any vulnerable or bad words then pattern classifier removes those words and these words stores in pattern classifier Man- ager.

\section{F. Authentication manager}

The Pattern classifier manager is responsible for capturing the whole transaction of the authentication and spam messages. You can check all the details regarding biometric authentication with their tags (Image-name, Date and time and status). The PCM can view the scanning report of spam message with their tags Filename, invalid words, Receivers and Date and time, and also can filter the fake injected data and captures in the attacker table with their tags File name, Injected data, Date and Time.

Fig1:- Proposed Methodlogy 


\section{A Secure Communication using Smart Mobile and Cloud Process}

\section{G. Receiver}

In this module, the Receivers (Reciever1, Reciever2, Reciever3, and Reciever4) can receive the file sent from the service provider via Pattern classifier.

\section{H. Threat model}

In this model, Attacker adds fake data when service provider wants to send a file to receivers, in the middle of service provider and pattern classifier. The Attacker may have chance to attack on file or he can inject a false data. And these attacked details are recognized by pattern classifiers. If injected data found then all these details are sent to Pattern classifier manager (PCM), after removing this injected data, file will be sent safely to respective receiver(Reciever1, Reciever2, Reciever3, and Reciever4).

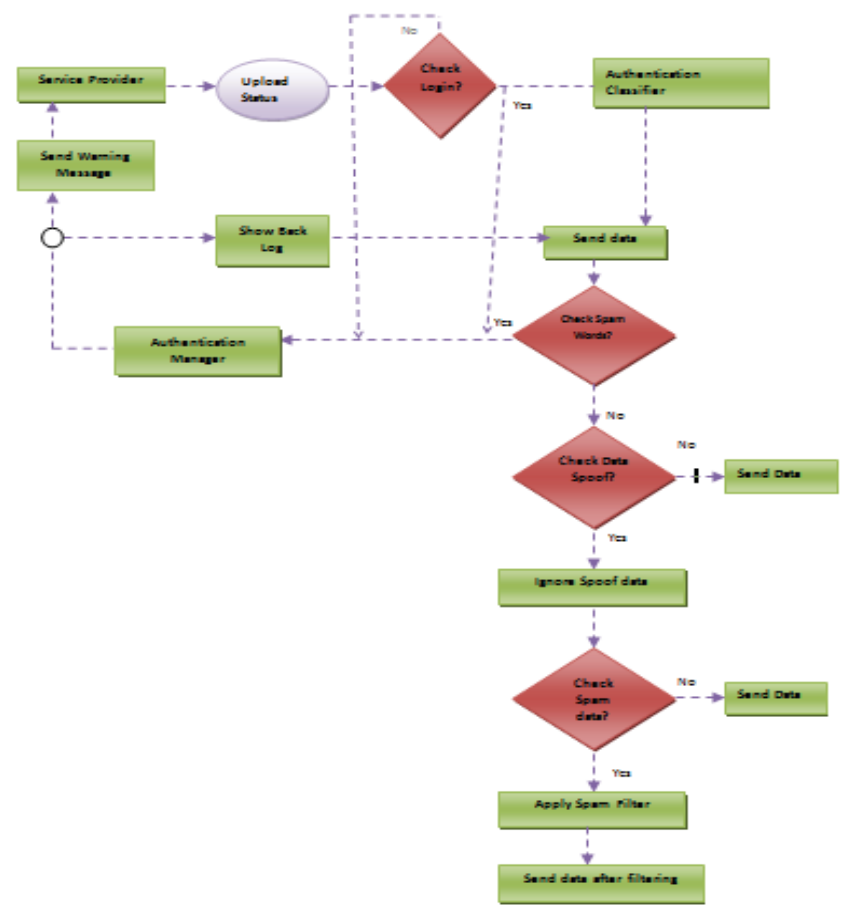

Fig 2: Flow Implementation of System

\section{RESULTS}

Security Analysis of Smartphone and Cloud Computing Authentication Frameworks and Protocols

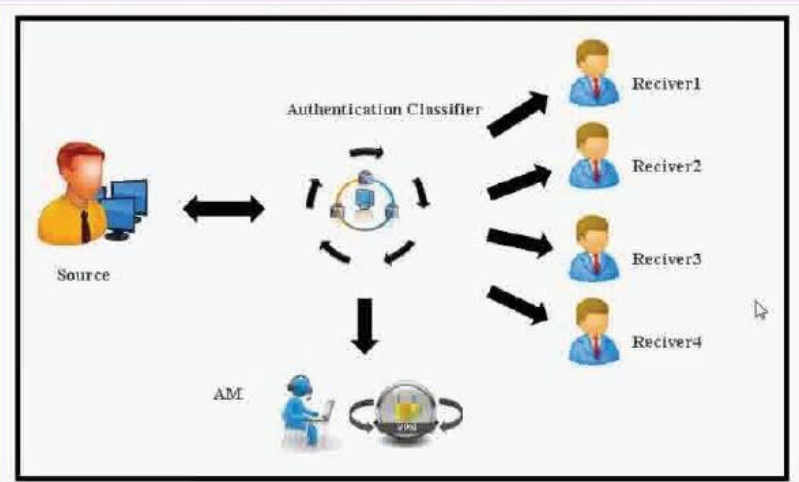

Fig 3: Simulator For Security Access
Security Analysis of Smartphone and Cloud Computing Authentication Frameworks and Protocols - - Authentication Manager

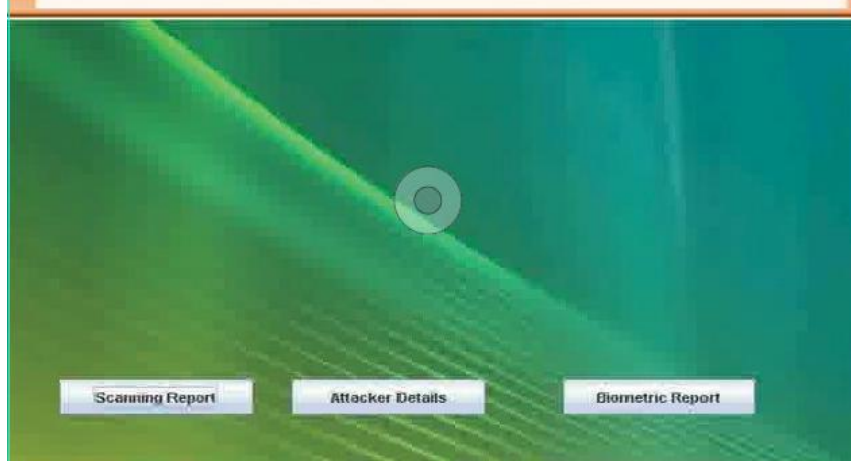

Fig 4 : Authentication Manger Analysis Window

Security Analysis of Smartphone and Cloud Computing

Authentication Frameworks and Protocols - Receiver

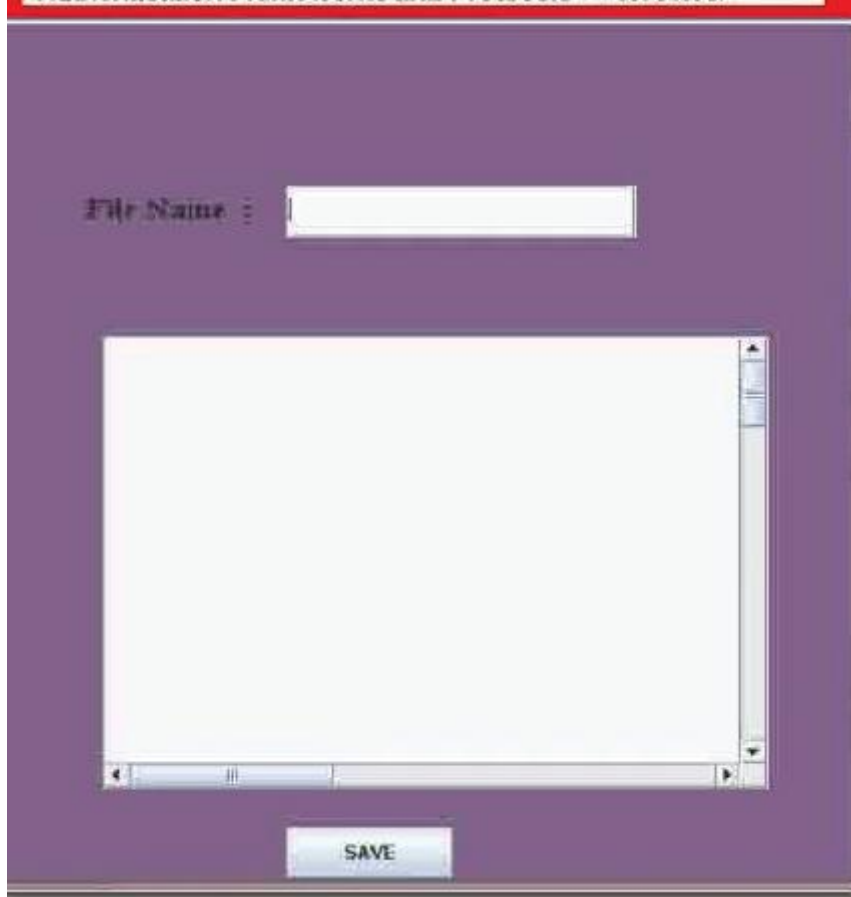

Fig 5:- Data Receiver Window

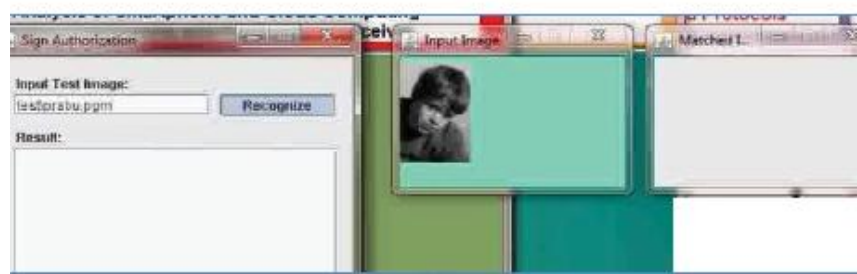

Fig 6 : Face authentication Process

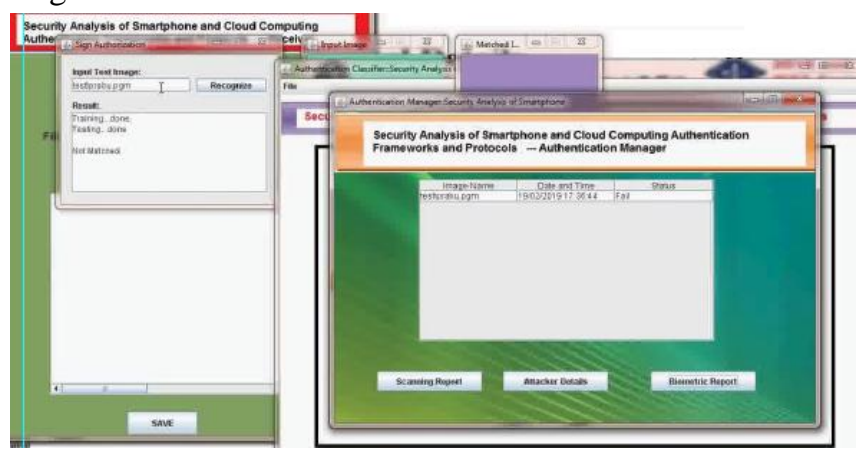

Fig7 : Face authentication Verification

Published By:

Blue Eyes Intelligence Engineering 


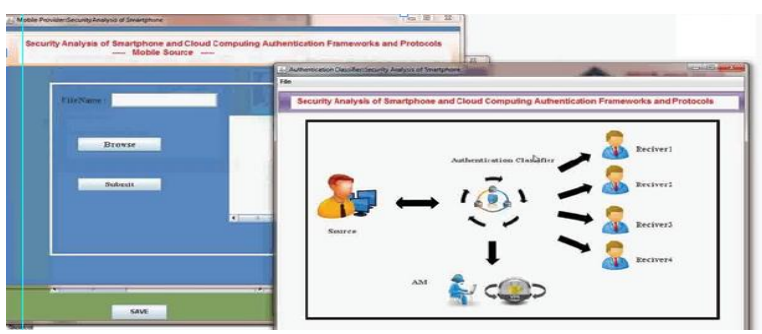

Fig 8: Sender sends data And Simulator Simulates Verification

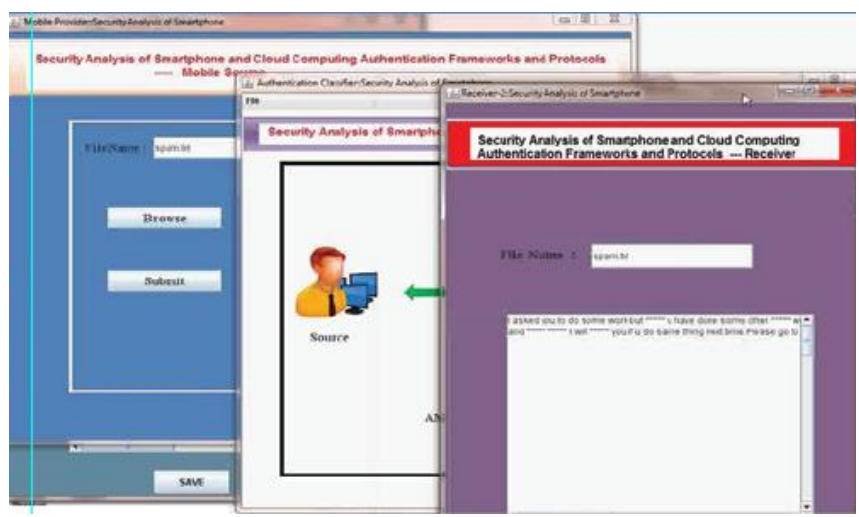

Fig 9: Receiver Side Data Receiving Process

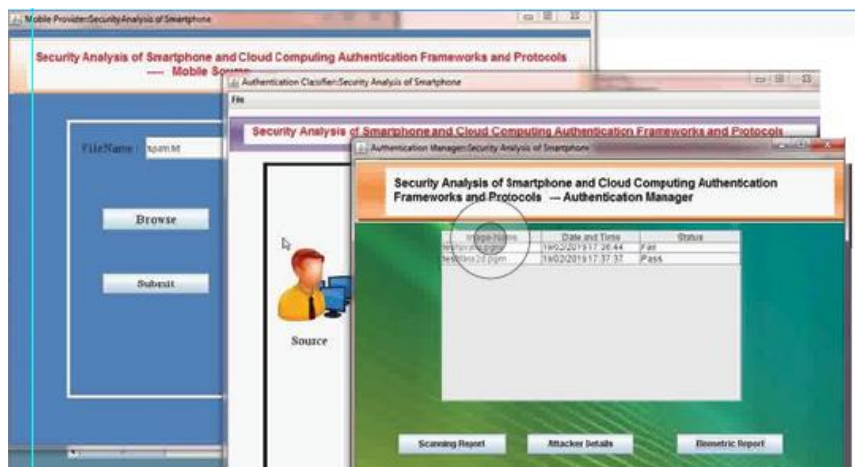

Fig 10: Evaluating Authentication Manager

\section{RESULT AND CONCLUSION}

The utilization of present day technologies, for example, Smartphones, have expanded the interest for more progressively secure, dependable and easy to use verification frameworks to encourage certified end-clients. Existing Smart-phone and Cloud Computing validation protocols and frame-works are vulnerable against various confirmation and security assaults. This examination has per- shaped a point by point audit of a few confirmation structures and conventions to framework and address numerous diligent security issues/imperfections and different impediments. The essential aim of the investigation was to abridge and feature security exposure and other disturbing issues to find the present cutting edge in the space. The security exposure and problems laid out will help with empowering the full and complete capability of 3Factor Authentication validation structures and conventions in Smart-phones and Cloud Computing situations. As the outcome of security blemishes and restrictions decided in the examination, plainly extra research work is required in various ways that think about the accompanying:

The coming of Smartphone has supplanted the utilization of smartcards from inside many do-mains. Be that as it may, multifaceted and 3Factor Authentication confirmation structures and Mechanisms are as yet untimely in present day Smartphones like Samsung mobiles and iPhone. Authentication is delicate and can without much of a stretch be avoided through a few ruptures. One of the problems was to disregard the re-validation of confirmation factors, while verifying expert seeding verification factors. For instance, after a fruit full Factor Authentication confirmation, the suggested validation conventions don't reverify

\section{Factor Authentication, while confirming}

Factor Authentication in the following verification stage. The greater part of the verification conventions are asset hungry and require a long keep running of validation circles. This dependably purposes an un expected postponement amid the verification procedure.

Numerous confirmation conventions, in the case of being suggested for Smartphones or $\mathrm{CC}$, are not created remembering their pertinent usage situations. A portion of the verification systems and conventions are not practical, when thinking about for useful execution. This is because of the way that the arrangements are impractical which requires unprecedented rebuilding. In various Cloud Computing verification structures and conventions, complete 3Fsarent actualized because of $\mathrm{t}$ h e confinements of the present cutting edge. Steadfastness of cloud benefits on outsider assets has made it sensitive to adjust and depend on.

\section{FUTURE ENHANCEMENTS}

Authentication protocols are usuallyconsidered to be resource heavy and require a long run of authentication-loops. Because of this there are usually unanticipated delays throughout the authentication-process. In future we propose to evaluate the time complexity and trust behavior.

\section{REFERENCES}

1) AbduIIah, A.,Siddiqui, Z, Khan, M. and A,AIghamdi,"Smart Environment as a Service: Three Factor CIoud Based User Authentication for TeIecare MedicaI lnformation System”. JournaI of MedicaI Systems. 2014. 38 (1):2-15.

2) The Statistics PortaI (2014), "Number of smartphone users wordlwide from 2014 to 2020", Extracted from https:// www.statista.com/statistics/330685/number-ofsmartphone-usersworIdwide/.

3) GlobaI Privacy Enforcement Network (2014), “75\% of Mobile Apps Want Access to User Data", Extracted from https:// www.futuristgerd.com/wpcontent/ upIoads/2015/04/chartoftheday_2710_App_Privacy.jpg.L.

4) FIynn and W. KIieber, "SmartphoneSecurity" in 1EEE Pervasive Computing, vol.16, number 7, page 17-22, Sept.-Nov. 2016. 
5) Dardanelli et aI., "A Security Layer for Smartphone-to-Vehicle Communication Over Bluetooth" in lEEE Embedded Systems Letters, volume 1, number 2, page 33-35, Sept. 2014.

6) J. K. Seifert,X. Zhung and P. Acllçmez, "Design and lmplementation of Efficient Integrity Protection for Open MobilePlatforms" in IEEE Transactions on Mobile Computing, volume 12, number 2, page 189-202, Feb. 2015.

7) P. Van Orschot and D. Karrera, "Secure Software InstaIIation on Smartphones" in IEEE Security \& Privacy, volume 8, number 4 , page44-49, May-June 2012.

8) V. Manilopoulos,S. Gosdakis, S. Toa, A. Rusk and P. Papadimtratos, "Secure and Privacy-Preserving Smartphone-Based Traffic Information Systems" in 1EEE Transactions on Intelligent Transportation Systems, volume 12, number 2, page 1422-1432, July 2015

9) Y. Fax, W. Tak, A. Ghineim,S. Dustar and M. A. Hossin, "From the Service-Oriented Architecture to the Web API Economy" in IEEE Internet Computing, volume 21, number 5,page 65-69, July-Aug. 2014

10) S. Zho,T. Zang, B. Lu, M. Marina, B. Chen and J. Cheng, "Lightweight SOA-based twin-engine architecture for enterprise systems in fixed and mobile environments" in China Communications, volume 12, number 8 , page188-199, Sept. 2015.

11) S. Rama,P. C. Hershely, C. B. Silio and A. Narayana, "System of Systems for Quality-of- Service Observation and Response in Cloud Computing Environments" in IEEE Systems Journal, volume 9, number 1, page 222-232, March 2016.

12) M. A. Sehani and A. Benarref, "Novel Cloud and SOA-Based Framework for E- Health Monitoring Using Wireless Biosensors" in IEEE Journal of Biomedical and Health Informatics, volume 19, number 2, page 47-59, March. 2015

13) Y. Yin, Q. Dun and A. V. Vasilakos, "A Survey on Service-Oriented Network Virtualization Toward Convergence of Networking and Cloud Computing" in 1EEE Transactions on Network and Service Management, volume 98, number 5, page 374-391, Dec 2011.

14) S. Burger et al., "Security intelligence for cloud management infrastructures" in 1BM JournaI of Research and Development, volume 61, number 5, page 21:2-21:23, Jun-Sept.2017.

15) M. Contl, C. A. Aradagna, M. Leone and J. Stella, "An Anonymous End-to- End Communication Protocol for Mobile Cloud Environments" in IEEE Transactions on Services Computing, volume 6, number 4, page 363-376, Jun- Aug. 2015.

16) D. De Figueiremo, "The Case for MobileTwo- Factor Authentication" in 1EEE Security \& Privacy, volume 8 , number 4 , page $82-84$, Oct.-Nov. 2012.

17) Nack Hun Kom, Young SiI Lee, Huotaek Lim, Hon Jae Lee and Heung Kuk Jo, "Online banking authentication system using mobile- OTP with QRcode" Computer Sciences and Convergence Information Technology (ICCIT), 2011 6th International Conference on, SeouI,2011, page 643-647.

18) P. Dale and K. Fulerud, "Secure and Inclusive Authentication with a Talking Mobile One-Time- Password Client" in 1EEE Security\& Privacy, volume8, number 1, page 37-44, Mar-Apr 2015 